dominant note has been that nothing should be done to harm the interests of the patients. This is a sound basis on which the profession can unite in insisting on obtaining for its junior members proper terms and conditions of service.

\section{Drugs Against Viruses}

At the present time antiviral chemotherapy is one of the most rapidly progressing branches of medical science. Drugs of proved efficacy are available commercially and are being used for the prevention and treatment of virus diseases of man. The viruses which have succumbed to the attack include smallpox (both variola major and alastrim), vaccinia, and herpes simplex, and the outlook is promising in the field of influenza and other virus infections of the respiratory tract.

The pioneer observation of the antiviral activity of benzaldehyde thiosemicarbazone and certain of its derivatives made by D. Hamre, J. Bernstein, and R. Donovick ${ }^{1}$ in 1950 led over the course of the years to the development of methisazone (Marboran) and its trial in Madras as a prophylactic agent against smallpox in 1963. ${ }^{2}$ The final results of this trial have recently been described by D. J. Bauer. ${ }^{3}$

Methisazone was given to 2,287 persons who had been in intimate contact with patients with smallpox. Among them six persons $(0.26 \%)$ subsequently developed smallpox, of whom two died. Among a group of 2,665 similarly exposed persons who were not treated, there were 105 cases (3.94\%), with 18 deaths. The reduction in the incidence of contact cases associated with methisazone treatment was highly significant. Persons who have been in contact with smallpox should be treated with methisazone in order to obtain immediate protection against the exposure which has already taken place, but they should also be vaccinated to confer protection against further exposure which may take place in the near future. The main side-effect of the drug was vomiting.

A similar protective effect of methisazone was also observed by Ribeiro do Valle and co-workers ${ }^{4}$ among persons exposed to alastrim infection in São Paulo, Brazil. Thus, among 384 contacts who received treatment with methisazone, mostly in lower doses than those used in the Madras trials, eight developed alastrim, whereas among 520 contacts who received no treatment there were 42 cases. Most of the contacts were not vaccinated or revaccinated after exposure (347 in the treatment group and 449 in the untreated group), so that methisazone in the absence of vaccination was an effective measure from the public-health point of view for reducing the incidence of contact cases and controlling an outbreak.

A prophylactic effect among persons who have been exposed to smallpox has also been obtained with $M$. and B. 7714 (3-methyl-4-bromo-5-formylisothiazole thiosemicarbazone). ${ }^{5}$ Among 196 contacts treated with the drug there were 40 cases

\footnotetext{
'Hamre, D., Bernstein, J., and Donovick, R., Proc. Soc. exp. Biol. (N.Y.), 1950, 73, 275.

Bauer, D. J., St. Vircent, L., Kempe, C. H., and Downie, A. W., Lancet, 1963, 2, 494. April International Congress of Infectious Diseases, Munich, 26-30

4 Ribeiro do Valle, L. A., Raposo de Melo, P., de Salles Gomes, L. F., and Morato Proença, L., Lancet, 1965, 2,'976.

Rao, A. R., McKendrick, G. D. W., Velayudhan, L., and Kamalakshi, K., ibid., 1966, 1, 1072.

- Bauer, D.' J., Ann N.Y. Acad. Sci., 1965, 130, 110.

'Adels, B. R., and Oppe, T. E., Lancet, 1966, 1, 18.

- Kaufman, H. E., Martola, E.-L., and Dohlman, C., Arch. Ophthal. 1962, 68, 235 .
}

of smallpox, compared with 60 cases among 201 contacts who were left untreated. The difference was significant at the $5 \%$ level. The authors state that $M$. and B. 7714 is not to be recommended in the prophylaxis of smallpox, and that the slightness of the prophylactic effect compared with that of methisazone cannot be ascribed to poor absorption as a consequence of vomiting. But further trial might be appropriate with smaller and more frequent doses in an attempt to reduce vomiting, for in the face of a life-threatening infection such as smallpox the free use of a drug with relatively unimportant side-effects is justifiable.

The lack of success in antiviral chemotherapy up to recent times can probably be ascribed to the fact that antiviral drugs have always been visualized as therapeutic agents. But the results obtained in India and Brazil show that their main application at present is in prophylaxis. The antiviral thiosemicarbazones can act only against virus which has entered cells and is actively multiplying. They therefore protect a smallpox contact by suppressing an infection which is already under way but which has not yet become clinically manifest. If the contact has escaped smallpox infection, then the drugs will naturally do nothing. Chemoprophylaxis with these antiviral agents is really therefore early treatment, but from the public-health point of view it seems justified to retain the term prophylaxis in this special sense because there is no way of telling which contacts are already in the incubation period except by awaiting events.

Some success has also been achieved in the treatment of other virus diseases. Bauer ${ }^{6}$ has reported recovery of patients with vaccinia gangrenosa treated with methisazone. Apparent benefit has also been obtained in the treatment of eczema vaccinatum, ${ }^{67}$ though the course of this disease is so variable that the effect of treatment is difficult to assess. It would seem desirable to investigate the effect of methisazone in this condition by means of a double-blind trial. In a preliminary communication this week in the B.M.F. (page 625) Dr. T. F. Sandeman, of Melbourne, reports that some patients with malignant lymphoma improved when given methisazone. Though it is too early to draw any firm conclusions from these results, they will be noted with interest.

Success in the treatment of virus diseases is not confined to the thiosemicarbazones. Idoxuridine ${ }^{8}$ is now the established drug for treating herpetic ulcers of the cornea, and it is also active against vaccinia infection. Other drugs which are in the process of development include amantidine hydrochloride, which has some prophylactic effect against influenza, and statolon, a substance which induces cells to manufacture interferon.

\section{Inappropriate Secretion of Antidiuretic Hormone}

A low concentration of sodium in the serum (hyponatraemia) is found in a variety of conditions. It may be associated with excessive loss of sodium in the urine, as in Addison's disease, when there is also hypotension and contraction of the volume of extracellular fluid. It may also occur in oedematous states, such as congestive cardiac failure and portal cirrhosis, in which urinary excretion of sodium is greatly reduced and there is expansion of the volume of extracellular fluid. In recent years a third type of hyponatraemia has been recognized in which, despite normal adrenal and kidney function, 
there is an excessive excretion of sodium so that the urine has a greater osmotic pressure than the plasma. Unlike the other two varieties, the patients do not suffer from dehydration, hypotension, or uraemia, and there is no clinical evidence of oedema. The condition has been attributed to inappropriate secretion of antidiuretic hormone (A.D.H.), ${ }^{1}$ because it resembles the state produced experimentally by administration of vasopressin to persons who have drunk large quantities of water, and who would normally pass large volumes of dilute urine. ${ }^{2}$ The low sodium level in the plasma is believed to be due to retention of water with resultant dilution of plasma solutes and expansion of the extracellular fluid: this leads to increased excretion of sodium and further decrease in the osmotic pressure of the plasma. Secretion of aldosterone is normally inhibited in these circumstances, but occasionally increased secretion may compensate for the sodium loss, though clearance of free water remains diminished and provides a reliable guide to the presence of the syndrome. ${ }^{3}$ No explanation has yet been found for the continued secretion of A.D.H. in spite of a hypotonic plasma.

Of conditions associated with inappropriate secretion of A.D.H. the two which have received most attention are bronchial carcinoma ${ }^{145}$ and intracranial disease. ${ }^{67}$ The syndrome has also been described in myxoedema, ${ }^{8}$ porphyria, ${ }^{9}$ and advanced tuberculosis, ${ }^{10}$ and in one patient episodes occurred over a period of five years without any clinical evidence of underlying disease. ${ }^{11}$ In nearly all the cases of bronchial carcinoma the growths were of the anaplastic oatcelled variety, so it seems unlikely that the tumour exerts its effect by mechanical interference with the volume receptors thought to be present in the mediastinum. The tumour may produce an A.D.H.-like compound or some substance which stimulates A.D.H. production. If this were the explanation, however, it would be expected that carcinomata in other sites would have the same effect, as with some of the other carcinomatous syndromes. Cerebral metastases are not the cause, although "dilutional hyponatraemia" and "cerebral salt wasting" have been described in cerebral trauma, encephalitis, pituitary tumours, and cerebrovascular disease. ${ }^{67}$ Here the mechanisms may more readily be explained by an irritant action on the hypothalamus and the posterior pituitary.

It is uncertain whether the hyponatraemia is responsible for any specific symptoms in this syndrome, though mental confusion and irritability have been attributed to it, and a general improvement in well-being may follow appropriate treatment. ${ }^{5}$ Some patients develop signs of water intoxication, with headaches and convulsions. Skin pigmentation has also been noted, and it is important to exclude the possibility of adrenocortical insufficiency or disorders of renal tubular function. Improvement may follow treatment of the underlying condition, but in the case of bronchial carcinoma this

\footnotetext{
Schwartz, W. B., Bennett, W., Curelop, S., and Bartter, F. C., Amer 7. Med., 1957, 23, 529 .

' Leaf, A., Bartter, F. C., Santos, R. F., and Wrong, O., f. clin. Invest., $1953,32,868$

3 Bernard-Weil, E., Schweiz. med. Wschr., 1966, 96, 212.

Amatruda, T. T., jun., Mulrow, P. J., Gallagher, J. C., and Sawyer, W. $H$, New Engl. 7 Med., 1963, 269, 544.

5 Ross, E. J., Quart. f. Med., 1963, 32, 297.

- Goldberg, M., and Handler, J. S., New Engl. f. Med., 1960, 263, 1037.

7 Epstein, F. H., Levitin, H., Glaser, G., and Lavietes, P., ibid., 1961, $265,513$.

- Goldberg, M., and Reivich, M., Ann. intern. Med., 1962, 56, 120.

- Nielsen, B., and Thorn, N. A., Amer. F. Med., 1965, 38, 345.

Sims, E. A. H., Welt, L. G., Orlof, J., and Needham, J. W., f. clin. Invest., 1950, 29, 1545 .

1 Grumer, H. A., Derryberry, W., Dubin, A., and Waldstein, S. S., Amer. F. Med., 1962, 32, 954.
}

is often only partially successful. It is sometimes possible to inhibit A.D.H. secretion by nicotine or alcohol, but this is unpleasant for the patient. Restriction of water intake may help, but administration of $9 \alpha$-fluorohydrocortisone is more effective and constitutes less of a hardship. ${ }^{5}$ The use of A.C.T.H. and corticosteroids has been generally unsatisfactory, but French workers ${ }^{3}$ have recently found that a slowrelease preparation of oxytocin together with small doses of A.C.T.H. restored free water clearance to normal, especially in patients with cerebral lesions.

\section{Aberdeen Typhoid Outbreak of 1964}

A factual record of the clinical, epidemiological, and publichealth aspects of the Aberdeen typhoid outbreak of $1964^{1}$ is of special interest, for the outbreak had several notable features. There were 507 cases, derived from 309 households in the city and 33 in the surrounding districts, with three deaths. All but four patients were treated in hospital ; the diagnosis was confirmed bacteriologically in 403 and clinically in 66 ; in the remaining 38 typhoid was not confirmed. Problems of the carrier state were discussed in these columns last week ${ }^{2}$ with reference to a report on the Aberdeen outbreak by Elizabeth M. Russell and her colleagues, ${ }^{3}$ while J. C. M. Sharp ${ }^{4}$ described the psychological disturbances that may result from it.

The outbreak appeared as one single wave and there were no proved secondary cases. The first patient, who became ill on 12 May 1964, was admitted to hospital on 16 May. A sample of blood submitted for bacteriological examination was reported positive for Salmonella typhi on 20 May, and four members from that family were immediately admitted to hospital. Since 38 of 41 patients admitted up to 23 May gave a history of having eaten cold meat purchased from the supermarket, and as three-quarters of them remembered eating sliced corned beef, suspicion centred on this article of food as the probable source. Strong support for this hypothesis was forthcoming when the Public Health Laboratory at Colindale reported that the organism from the first isolations was phage type 34, a variety common in South America and Spain, but virtually unknown in Great Britain. Up to 26 May the outbreak seemed to be confined to persons who had eaten corned beef from a single large tin which had been sold in slices between 6 and 9 May. Thereafter the picture changed and more and more cases appeared with a history of having eaten other cold meats from the supermarket. It then became apparent that the slicing machine used for the corned beef had infected other cold meats on 8 and 9 May and that the organisms had multiplied when the lightly contaminated meats had been stored in an uncooled display window exposed to sunlight. The Health and Welfare Department took action to cleanse the supermarket between 20 and 23 May and this coincided with complete cessation of spread from that source. Intensive investigation failed to reveal a carrier among the staff nor had any member ever visited an area in which phage type 34 had been found. The " all clear" was given on 17 June, 28 days after the first notification of suspects. In all, 391 patients had given a history of eating food from the infected shop, 373 having eaten cold meats and 18 fruit.

This epidemic of typhoid, the largest in Britain since 1937, contains many lessons of importance. Epidemiologically, the 